\title{
Molecular Structure of Atomic Nucleus
}

\author{
Vu B. Ho \\ Advanced Study, 9 Adela Court, Mulgrave, Victoria, Australia \\ Email: vubho@bigpond.net.au
}

How to cite this paper: Ho, V.B. (2020) Molecular Structure of Atomic Nucleus. Journal of Modern Physics, 11, 1395-1409. https://doi.org/10.4236/jmp.2020.119087

Received: August 30, 2020

Accepted: September 20, 2020

Published: September 23, 2020

Copyright (c) 2020 by author(s) and Scientific Research Publishing Inc. This work is licensed under the Creative Commons Attribution International License (CC BY 4.0).

http://creativecommons.org/licenses/by/4.0/

\begin{abstract}
In this work, we extend our work on the Heisenberg model of the neutron formulated as a dwarf hydrogen-like atom under the influence of the More General Exponential Screened Coulomb Potential (MGESCP) to show that an atomic nucleus may possess a molecular structure made up of atoms bonding together by a potential used to describe the strong force associated with a generalised Yukawa MGESCP potential. We show that the neutrons and protons are arranged into narrow lattices therefore they may fold to form threedimensional shells by bonding similar to hydrogen bonding. In particular, the nucleons may form stable structures such as that of fullerenes in which the vertices are occupied by the nucleons which are simply just protons. For example, a nucleus with a total number of 60 nucleons may arrange itself into the topological structure of a buckminsterfullerene. We also apply Schrödinger wave equation with central field approximation to describe the quantum dynamics of nuclei of atomic atoms that now possess the physical structure of a dwarf molecular ion.
\end{abstract}

\section{Keywords}

Heisenberg Model of the Neutron, Dwarf Hydrogen Atom, Molecular Structure of Atomic Nucleus, Weak and Strong Interactions, Yukawa MGESCP Potential, Fullerene

\section{Introduction}

In our work on quantum dynamics of Heisenberg model of the neutron associated with beta decay, we showed that a neutron may have the structure of a dwarf hydrogen-like atom and the beta decay is a physical process that requires a sophisticated form of potential for its dynamical description, such as a generalised Yukawa MGESCP potential. We also showed that this complicated process could be broken down into various consecutive stages of physical processes each of which requires a different form of potential for its mathematical formulation. 
Now, it is anticipated that the view of the neutron as a dwarf hydrogen-like atom would lead to different perspectives on physical structures of an atomic nucleus. In this work, we will discuss one such implication and show that the view of the neutron as a dwarf hydrogen-like atom provides the construction of a new model for atomic nucleus in which the nucleus would possess the structure of a molecule made up of atoms bonding together and in particular the bonding can be described using the form of a potential that is used to describe the strong force. Specifically, we show that the molecular structure of an atomic nucleus can be constructed similarly to the molecular structure of the hydrogen molecule $\mathrm{H}_{2}$ composed of two hydrogen atoms each of which has one proton and one electron, and the corresponding molecular ion $\mathrm{H}_{2}^{+}$which has only one electron. Despite the neutral molecule $\mathrm{H}_{2}$ has two protons and two electrons, the pair electrons are shared between the two protons to form the covalent bonding in which the electrons orbit both protons. Then, if one electron is removed from the neutral atomic molecule $\mathrm{H}_{2}$ we obtain the molecular ion $\mathrm{H}_{2}^{+}$which has only one electron which also orbits both protons. As discussed in Section 3, this picture of the molecular ion $\mathrm{H}_{2}^{+}$is the representation of the molecular structure that we will apply to the deuteron which is the nucleus of a deuterium composing of one proton and one neutron. However, instead of the usual Coulomb potential, we will assume that the electron and the two protons attract each other by a strong force which can be derived from a generalised Yukawa MGESCP potential. In Section 4, we will then extend our discussion into nuclei composed of many protons and neutrons and show how these nuclei can be formed to possess a molecular structure if the neutrons are regarded as dwarf hydrogen atoms. With regards to mathematical formulation, we show that it is possible to apply the central field approximation to describe the quantum dynamics of these molecules. However, for its foundational basis and a more complete presentation of the view that an atomic nucleus can be described as a molecule, we will give a brief account in the next section, the Heisenberg model of the neutron as dwarf hydrogen-like atom.

\section{Heisenberg Model of the Neutron as a Dwarf Hydrogen Atom}

In this section we outline the Heisenberg model of the neutron as dwarf hydrogen-like atom in which the bound states of a dwarf hydrogen atom can be formulated by using a general form of Yukawa potential known as the More General Exponential Screened Coulomb Potential (MGESCP) given as follows

$$
V(r)=-\frac{V_{0} \mathrm{e}^{-2 \alpha r}}{r}-\frac{V_{0}}{r}-V_{0} \alpha \mathrm{e}^{-2 \alpha r},
$$

where $V_{0}$ is the potential depth and the parameter $\alpha$ is the strength coupling constant [1] [2] [3]. In particular, the MGESCP potential can be reduced to the potential that has been proposed for the interactions between the quarks for strong force in particle physics and this result leads to an unexpected implication 
that a proton and an electron may also interact strongly at short distances. It is also worth mentioning here that there are prominent features that emerge from using the MGESCP potential to describe a neutron as a dwarf hydrogen atom, such as the energy spectrum of the bound states is continuous with respect to distance, and the Yukawa potential can be restrained to generate and determine mathematical structures of physical objects that may be identified with the quantum mediators associated with the weak and strong interactions. With this regard, it is reasonable to suggest that functional potentials in physics may have physical mechanisms to generate mediators of associated physical fields, and these mechanisms can be formulated in terms of differentiable manifolds and their corresponding direct sums of prime manifolds as discussed in our works on the possibility to formulate physics purely in terms of differential geometry and topology [4]. The time-independent Schrödinger equation of wave mechanics for a neutron as a dwarf hydrogen-like atom can be written as follows [5] [6]

$$
-\frac{\hbar^{2}}{2 \mu} \nabla^{2} \psi(\boldsymbol{r})+V(\boldsymbol{r}) \psi(\boldsymbol{r})=E \psi(\boldsymbol{r}),
$$

in which $V(\boldsymbol{r})$ is the MGESCP potential given in Equation (1). Since the MGESCP potential is spherically symmetric, Equation (2) can be written in the spherical polar coordinates as

$$
-\frac{\hbar^{2}}{2 \mu}\left(\frac{1}{r^{2}} \frac{\partial}{\partial r}\left(r^{2} \frac{\partial}{\partial r}\right)-\frac{\boldsymbol{L}^{2}}{\hbar^{2} r^{2}}\right) \psi(\boldsymbol{r})+\left(-\frac{V_{0}}{r}\left(1+(1+\alpha r) \mathrm{e}^{-2 \alpha r}\right)\right) \psi(\boldsymbol{r})=E \psi(\boldsymbol{r}),
$$

where the orbital angular momentum operator $\boldsymbol{L}^{2}$ is given by

$$
\boldsymbol{L}^{2}=-\hbar^{2}\left(\frac{1}{\sin \theta} \frac{\partial}{\partial \theta}\left(\sin \theta \frac{\partial}{\partial \theta}\right)+\frac{1}{\sin ^{2} \theta} \frac{\partial^{2}}{\partial^{2} \phi}\right) .
$$

Solutions of Equation (3) can be found using the separable form

$$
\psi_{n l}(\boldsymbol{r})=R_{n l}(r) Y_{l m}(\theta, \phi),
$$

where $R_{n l}$ is a radial function and $Y_{l m}$ is the spherical harmonic. Then Equation (3) is reduced to the system of equations

$$
\begin{gathered}
\boldsymbol{L}^{2} Y_{l m}(\theta, \phi)=l(l+1) \hbar^{2} Y_{l m}(\theta, \phi), \\
\left(-\frac{\hbar^{2}}{2 \mu}\left(\frac{\mathrm{d}^{2}}{\mathrm{~d} r^{2}}+\frac{2}{r} \frac{\mathrm{d}}{\mathrm{d} r}\right)+\frac{l(l+1) \hbar^{2}}{2 \mu r^{2}}-\frac{V_{0}}{r}\left(1+(1+\alpha r) \mathrm{e}^{-2 \alpha r}\right)\right) R_{n l}(r)=E R_{n l}(r) .
\end{gathered}
$$

It has been shown that the radial solution $R_{n l}(r)$ to Equation (7) can be obtained as

$$
R_{n l}(r)=N_{n l} r^{(-1+\sqrt{4 l(l+1)+1}) / 2} \mathrm{e}^{-\beta r} L_{n}^{1+\sqrt{4 l(l+1)+1}}(2 \beta r),
$$

and the corresponding energy spectrum $E_{n l}$ is given by

$$
E_{n l}=-V_{0} \alpha \mathrm{e}^{-2 \alpha r}-\frac{\mu}{2 \hbar^{2}}\left(\frac{\left(V_{0}+V_{0} \mathrm{e}^{-2 \alpha r}\right)}{n+l+1}\right)^{2},
$$


where $\beta^{2}=\left(2 \mu\left(V_{0}+V_{0} \mathrm{e}^{-2 \alpha r}\right) / \hbar^{2}(2 n+1+\sqrt{4 l(l+1)+1})\right)^{2}$. Although this energy spectrum is discrete with respect to the quantum numbers $n$ and $l$, it depends continuously on the radial distance $r$. In order to interpret the energy spectrum given in Equation (9) as some energy spectrum associated with the beta minus decay we need to apply the restraint condition applied to the Yukawa potential so that the MGESCP potential is reduced to the potential that is used to describe strong interaction at very short distances so that a proton and an electron can form a dwarf hydrogen-like atom. In fact, we showed in our work on a quantum dynamics of beta decay that if the Yukawa potential remains constant, i.e., $\mathrm{e}^{-m r} / r=k J / 2 a$, where $m$ and $k J$ are parameters in the generalised Dirac equation given in the form [7] [8]

$$
\gamma^{\mu} \partial_{\mu} \psi=-i m \psi+i k J
$$

then the generalised Yukawa MGESCP potential given in Equation (2) is reduced to the potential that is used to describe the strong force.

$$
\begin{aligned}
V(r) & =-\frac{V_{0}}{r}\left(1+(1+\alpha r) \mathrm{e}^{-2 \alpha r}\right) \\
& =-\frac{V_{0}}{r}+\frac{k J}{2 \alpha}(1+\alpha r) \\
& =-\frac{V_{0}}{r}+\frac{k J}{2} r+\frac{k J}{2 \alpha}
\end{aligned}
$$

And from the energy spectrum given in Equation (9) we also obtain

$$
E_{n l}=-\frac{k J}{2} r-\frac{\mu_{e}}{2 \hbar^{2}}\left(\frac{\left(V_{0}+(k J \alpha / 2) r\right)}{n+l+1}\right)^{2}
$$

Now we may interpret this continuous spectrum of energy with respect to distance as the energy spectrum of massive mediators associated with strong force described by the potential given in Equation (11). When a physical particle is created it is being created continuously until it reaches the size that is required for the system. This process happens in a very short time therefore it seems like an instantaneous creation. In particle physics, the parameter $\alpha$ of the exponential term is expressed in terms of the mass $m$ of a force carrier as $\alpha=m c / 2 \hbar$. Therefore when the mass of the force carrier is being continuously created the parameter $\alpha$ is being getting larger, at the same time the radius $r$ is also getting bigger, therefore the term $\mathrm{e}^{-2 \alpha r} \rightarrow 0$ and also the term $\alpha \mathrm{e}^{-2 \alpha r} \rightarrow 0$. The mass that is accumulated by the force carrier should be supplied by the neutron. When the force carrier with required mass hits the electron, the latter will move further from the proton. On the other hand the MGESCP potential is reduced to the mixed Coulomb-Yukawa potential when the process of creation of the force carrier is complete. This form of potential provides a repulsive force to move the electron away. It is also noted that the form of the potential given in Equation (1) is a specific form of a more general form of potential which can be written as

$$
V(r)=-\alpha \frac{\mathrm{e}^{-\beta r}}{r}+\frac{Q}{r}+K \mathrm{e}^{-\gamma r}
$$


where $K, Q, \alpha, \beta$ and $\gamma$ are physical parameters that will need to be determined. This potential would describe a more complete physical process of beta decay when it is applied to the Schrödinger wave equation given in Equation (2). However, whether the Schrödinger wave equation with the form of potential given in Equation (13) could be solved to obtain exact solutions similar to solutions given in Equation (8) requires more rigorous mathematical investigations.

\section{Molecular Structure of the Nucleus of a Deuterium}

In this section we discuss a molecular structure for the nucleus of a deuterium with regard to the view that a neutron is formulated as a dwarf hydrogen-like atom. We have two different formations that can be applied to the physical structure of the deuterium described as follows. If the Coulomb potential is applied, then a deuterium is an isotope of the normal hydrogen atom with a neutron in the nucleus besides the proton, as shown in Figure 1. However, if a generalised Yukawa MGESCP potential is applied then a deuterium may be viewed as an isotope of the helium atom without neutrons at the nucleus but only two protons and two electrons one of which can be supposed to be orbiting very close to the two protons, as shown in Figure 2.

In the following we will examine how the two different formations of the deuteron and the deuterium, as depicted above, and their corresponding quantum dynamics can be formulated using Schrödinger wave equation in quantum mechanics. For the case of the deuteron, which is the nucleus of the deuterium as shown in Figure 1, it has been shown from experiments that the bound state of the deuteron exists in a triplet state that can be described by the Schrödinger wave equation in the centre-of-mass system as

$$
\left(-\frac{\hbar^{2}}{2 \mu} \nabla^{2}+V(r)-E\right) \psi(\boldsymbol{r})=0
$$

where $\mu=m_{p} m_{n} /\left(m_{p}+m_{n}\right)$ and the potential $V(r)$ can be taken as a square well potential $\left\{V(r)=-V_{0}\right.$ for $r<a$ and $V(r)=0$ for $\left.r>a\right\}$, or a Yukawa potential $\left\{V(r)=-V_{0} r^{-1} \exp (-r / a)\right\}$, or an exponential potential $\left\{V(r)=-V_{0} \exp (-r / a)\right\}$ [9].

However, if the deuteron has a physical structure as shown in Figure 2 then it can also be viewed as a molecular ion which is composed of two protons and one electron, and the physical system is described by using a generalised Yukawa potential rather than the usual Coulomb potential as for the case of the hydrogen molecular ion $\mathrm{H}_{2}^{+}$. If we assume the generalised Yukawa potential to be the MGESCP potential given in Equation (1), i.e., $V(r)=-V_{0} \mathrm{e}^{-2 \alpha r} / r-V_{0} / r-V_{0} \alpha \mathrm{e}^{-2 \alpha r}$, and using the centre-of-mass coordinates for the deuteron molecular ion, as shown in Figure 3, where $\boldsymbol{R}$ is the relative position vector of the two protons positioned at $A$ and $B ; \quad r$ is the position vector of the electron at $C ; r_{A}$ and $r_{B}$ are the distances of the electron from the protons, then the Schrödinger wave equation for the deuteron molecular ion can be written as 


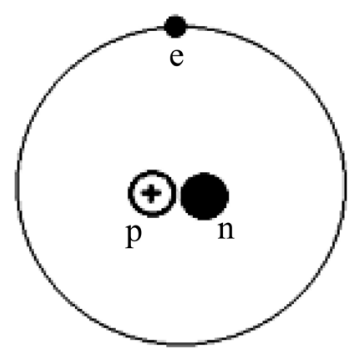

Figure 1. Deuterium with Coulomb potential.

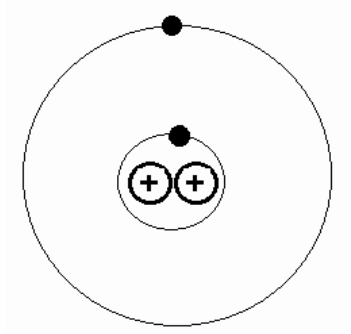

Figure 2. Deuterium with generalised Yukawa potential.

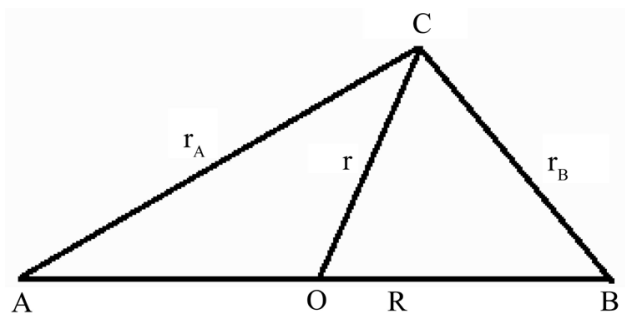

Figure 3. The centre-of-mass coordinate system for deuteron molecular ion.

$$
\begin{aligned}
& \left(-\frac{\hbar^{2}}{2 \mu_{A B}} \nabla_{R}^{2}-\frac{\hbar^{2}}{2 \mu_{e}} \nabla_{r}^{2}-\frac{V_{0} \mathrm{e}^{-2 \alpha r_{A}}}{r_{A}}-\frac{V_{0}}{r_{A}}-V_{0} \alpha \mathrm{e}^{-2 \alpha r_{A}}\right. \\
& \left.-\frac{V_{0} \mathrm{e}^{-2 \alpha r_{B}}}{r_{B}}-\frac{V_{0}}{r_{B}}-V_{0} \alpha \mathrm{e}^{-2 \alpha r_{B}}+\frac{q^{2}}{4 \pi \varepsilon_{0} R}-E\right) \psi(\boldsymbol{r}, \boldsymbol{R})=0
\end{aligned}
$$

where $\mu_{A B}=m_{p} / 2$ and $m_{p}$ is the mass of the proton; and the reduced mass $\mu_{e}$ of the electron is given as $\mu_{e}=2 m m_{p} /\left(m+2 m_{p}\right) \approx m$ where $m$ is the mass of the electron. In the Born-Oppenheimer approximation the wave motion of the electron described by a new wavefunction $\chi_{j}(\boldsymbol{r}, \boldsymbol{R})$ that satisfies the following equation [10]

$$
\begin{aligned}
& \left(-\frac{\hbar^{2}}{2 \mu_{e}} \nabla_{r}^{2}-\frac{V_{0} \mathrm{e}^{-2 \alpha r_{A}}}{r_{A}}-\frac{V_{0}}{r_{A}}-V_{0} \alpha \mathrm{e}^{-2 \alpha r_{A}}-\frac{V_{0} \mathrm{e}^{-2 \alpha r_{B}}}{r_{B}}\right. \\
& \left.-\frac{V_{0}}{r_{B}}-V_{0} \alpha \mathrm{e}^{-2 \alpha r_{B}}+\frac{q^{2}}{4 \pi \varepsilon_{0} R}-E\right) \chi_{j}(\boldsymbol{r}, \boldsymbol{R})=0
\end{aligned}
$$

If the wave equation given in Equation (16) could be solved exactly then the wavefunctions $\chi_{j}(\boldsymbol{r}, \boldsymbol{R})$ would become the molecular orbitals of the deuteron 
in analogy with the atomic orbitals. In this case the eigenenergies $E_{j}(R)$ that correspond to the wavefunction $\chi_{j}(\boldsymbol{r}, \boldsymbol{R})$ would be found for each value of the distance $R$.

Now, it is also seen from Figure 2 that the physical structure of a deuterium also looks similar to that of a helium except there are no neutrons inside the nucleus and the quantum dynamics of the two electrons are described by two different potentials in which the inner electron is described by a generalised $\mathrm{Yu}$ kawa MGESCP potential and the outer electron simply by the Coulomb potential. In the following we will formulate this atomic structure of the deuterium using also the Schrödinger wave equation in quantum mechanics.

As shown in Figure 4, the Hamiltonian for a helium atom in which the two electrons under the influence of the Coulomb potential due to the two protons in the nucleus can be found to be of the form

$$
H=-\frac{\hbar^{2}}{2 \mu_{e}}\left(\nabla_{1}^{2}+\nabla_{2}^{2}\right)-\frac{2 q^{2}}{4 \pi \varepsilon_{0} r_{1}}-\frac{2 q^{2}}{4 \pi \varepsilon_{0} r_{2}}+\frac{q^{2}}{4 \pi \varepsilon_{0} r_{12}}
$$

where $\boldsymbol{r}_{1}$ and $\boldsymbol{r}_{2}$ are the positions of the two electrons and $r_{12}=\left|\boldsymbol{r}_{1}-\boldsymbol{r}_{2}\right|$. Since the distance $r_{12}$ between the two electrons in assumed to be short therefore the term $q^{2} / 4 \pi \varepsilon_{0} r_{12}$ is not negligible and the Schrödinger wave equation that is obtained from the Hamiltonian given in Equation (17) cannot be solved to obtain exact solutions. On the other hand, if we refer again to Figure 2 for the case of the deuterium in which the neutron is instead regarded as a dwarf hydrogen atom then the deuterium also has the atomic structure similar to the helium given in Figure 4, except the quantum dynamics of the outer electron is described by the Coulomb potential but the inner electron by the generalised Yukawa MGESCP potential given in Equation (1). The Hamiltonian in this case takes the form

$$
H=-\frac{\hbar^{2}}{2 \mu_{e}}\left(\nabla_{1}^{2}+\nabla_{2}^{2}\right)-\frac{2 q^{2}}{4 \pi \varepsilon_{0} r_{1}}-\frac{2 V_{0} \mathrm{e}^{-2 \alpha r_{2}}}{r_{2}}-\frac{2 V_{0}}{r_{2}}-2 V_{0} \alpha \mathrm{e}^{-2 \alpha r_{2}}+\frac{q^{2}}{4 \pi \varepsilon_{0} r_{12}}
$$

It is observed now that for the case to the deuterium as shown in Figure 2 the two electrons are on different orbits which are far away from each other therefore the last term in the Hamiltonian given in Equation (18) that involves the interaction between them is negligible due to large distance. In this case the Hamiltonian given in Equation (18) is reduced to

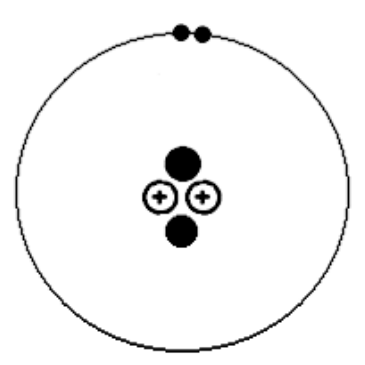

Figure 4. Helium with Coulomb potential. 


$$
H=-\frac{\hbar^{2}}{2 \mu_{e}} \nabla_{1}^{2}-\frac{2 q^{2}}{4 \pi \varepsilon_{0} r_{1}}-\frac{\hbar^{2}}{2 \mu_{e}} \nabla_{2}^{2}-\frac{2 V_{0} \mathrm{e}^{-2 \alpha r_{2}}}{r_{2}}-\frac{2 V_{0}}{r_{2}}-2 V_{0} \alpha \mathrm{e}^{-2 \alpha r_{2}}
$$

The corresponding Schrödinger wave equation is written as

$$
\left(-\frac{\hbar^{2}}{2 \mu_{e}} \nabla_{1}^{2}-\frac{2 q^{2}}{4 \pi \varepsilon_{0} r_{1}}-\frac{\hbar^{2}}{2 \mu_{e}} \nabla_{2}^{2}-\frac{2 V_{0} \mathrm{e}^{-2 \alpha r_{2}}}{r_{2}}-\frac{2 V_{0}}{r_{2}}-2 V_{0} \alpha \mathrm{e}^{-2 \alpha r_{2}}-E\right) \psi\left(\boldsymbol{r}_{1}, \boldsymbol{r}_{2}\right)=0(20)
$$

Since the Hamiltonian given in Equation (19) is the sum of two separate Hamiltonians therefore the corresponding wavefuntion that describes the system is separable and can be written as $\psi\left(\boldsymbol{r}_{1}, \boldsymbol{r}_{2}\right)=\psi_{1}\left(\boldsymbol{r}_{1}\right) \psi_{2}\left(\boldsymbol{r}_{2}\right)$. Using the separable wavefunction $\psi\left(\boldsymbol{r}_{1}, \boldsymbol{r}_{2}\right)=\psi_{1}\left(\boldsymbol{r}_{1}\right) \psi_{2}\left(\boldsymbol{r}_{2}\right)$ the Schrödinger wave equation given in Equation (20) can now be written as a system of two separate Schrödinger wave equations as follows

$$
\begin{gathered}
\left(-\frac{\hbar^{2}}{2 \mu_{e}} \nabla_{1}^{2}-\frac{2 q^{2}}{4 \pi \varepsilon_{0} r_{1}}\right) \psi_{1}\left(\boldsymbol{r}_{1}\right)=E_{1} \psi_{1}\left(\boldsymbol{r}_{1}\right) \\
\left(-\frac{\hbar^{2}}{2 \mu_{e}} \nabla_{2}^{2}-\frac{2 V_{0} \mathrm{e}^{-2 \alpha r_{2}}}{r_{2}}-\frac{2 V_{0}}{r_{2}}-2 V_{0} \alpha \mathrm{e}^{-2 \alpha r_{2}}\right) \psi_{2}\left(\boldsymbol{r}_{2}\right)=E_{2} \psi_{2}\left(\boldsymbol{r}_{2}\right)
\end{gathered}
$$

where $E=E_{1}+E_{2}$. Solutions to Equation (21) are the normalised radial eigenfunctions of the bound states that can be found and given as

$$
R_{n l}(r)=-\left(\left(\frac{2 k m q^{2}}{\hbar^{2} n}\right)^{3} \frac{(n-l-1) !}{2 n((n+l) !)^{3}}\right)^{1 / 2} \mathrm{e}^{-\rho / 2} \rho^{l} L_{n+l}^{2 l+1}(\rho)
$$

where $\rho=\left(-8 \mu_{e} E / \hbar^{2}\right)^{1 / 2} r$ and $L_{n+l}^{2 l+1}(\rho)$ is the associated Laguerre polynomial. The bound state energy eigenvalues are given by

$$
E_{n}=-\frac{\mu_{e} k^{2} q^{4}}{2 \hbar^{2}} \frac{1}{n^{2}}
$$

On the other hand, solutions to Equation (22) are the radial eigenfunctions of the bound states which can also be obtained and given in Equation (8), and the corresponding energy spectrum $E_{n l}$ is given in Equations (9) and (12) in Section 2.

\section{Molecular Structure of Nuclei Composed of Many Protons and Neutrons}

In this section we further discuss how a nucleus which is composed of many protons and neutrons can be formed to also possess a molecular structure if the neutrons are regarded as dwarf hydrogen atoms. In order to be able construct nuclei with many nucleons as molecules from the assumption that the neutron has the structure of a dwarf hydrogen atom we now assume further that the protons and the neutrons are arranged so that they will have the physical structure similar to that of a hydrogen molecule $\mathrm{H}_{2}$ or that of a hydrogen molecular ion $\mathrm{H}_{2}^{+}$. This means that they are arranged in a manner in which they can share electrons so that each of the nucleons is seen to have one or two electrons orbit- 
ing around it. As illustrations, in the following we give such possible structures for the first few nuclei, where $\oplus$ representing a neutron and $\oplus$ a proton. For nuclei with even numbers of protons and neutrons, first consider the case of the alpha particle which is the nucleus of the helium atom as shown in Figure 5.

In Figure 5, the two neutrons are assumed to share a pair of electrons described by the form of covalent bonding. On the other hand, each proton is assumed to receive an electron from each neutron. Overall, the two electrons from two neutrons are shared by the four protons in the nucleus. In this case the nucleus of the helium behaves like a solid in which the four protons are kept in place by the force of attraction between them and the electrons. The force of attraction in this case can be assumed to be the strong force between the electrons and the protons rather than the Coulomb force as in the case of a normal solid.

We will now formulate an approximate quantum description for this molecular structure of the atomic nucleus of a helium atom, as shown in Figure 6, using also the Schrödinger wave equation in quantum mechanics.

The Hamiltonian for a helium nucleus in which the two electrons are under the influence of the generalised Yukawa MGESCP potential given in Equation (1) due to the four protons in the nucleus can be written in the form

$$
\begin{aligned}
H= & -\frac{\hbar^{2}}{2 \mu_{e}}\left(\nabla_{1}^{2}+\nabla_{2}^{2}\right)-\frac{2 V_{0} \mathrm{e}^{-2 \alpha r_{1}}}{r_{1}}-\frac{2 V_{0}}{r_{1}}-2 V_{0} \alpha \mathrm{e}^{-2 \alpha \eta_{1}} \\
& -\frac{2 V_{0} \mathrm{e}^{-2 \alpha r_{2}}}{r_{2}}-\frac{2 V_{0}}{r_{2}}-2 V_{0} \alpha \mathrm{e}^{-2 \alpha r_{2}}+\frac{q^{2}}{4 \pi \varepsilon_{0} r_{12}}
\end{aligned}
$$

where $\boldsymbol{r}_{1}$ and $\boldsymbol{r}_{2}$ are the positions of the two electrons and $r_{12}=\left|\boldsymbol{r}_{1}-\boldsymbol{r}_{2}\right|$. We may now argue that under the influence of the generalised Yukawa MGESCP potential given in Equation (1) the electrons are attracted strongly to the protons in the nucleus therefore the attraction between the two electrons under the Coulomb force is much weaker therefore the last term in the Hamiltonian given in Equation (25) that involves the interaction between the two electrons is negligible, then we obtain

$$
\begin{aligned}
H= & -\frac{\hbar^{2}}{2 \mu_{e}}\left(\nabla_{1}^{2}+\nabla_{2}^{2}\right)-\frac{2 V_{0} \mathrm{e}^{-2 \alpha \eta_{1}}}{r_{1}}-\frac{2 V_{0}}{r_{1}}-2 V_{0} \alpha \mathrm{e}^{-2 \alpha r_{1}} \\
& -\frac{2 V_{0} \mathrm{e}^{-2 \alpha r_{2}}}{r_{2}}-\frac{2 V_{0}}{r_{2}}-2 V_{0} \alpha \mathrm{e}^{-2 \alpha r_{2}}
\end{aligned}
$$

Since the Hamiltonian given in Equation (26) is the sum of two separate Hamiltonians therefore the corresponding wavefuntion that describes the system is separable and can be written as $\psi\left(\boldsymbol{r}_{1}, \boldsymbol{r}_{2}\right)=\psi_{1}\left(\boldsymbol{r}_{1}\right) \psi_{2}\left(\boldsymbol{r}_{2}\right)$. The corresponding Schrödinger wave equation applied to the system takes the form

$$
\begin{aligned}
& \left(-\frac{\hbar^{2}}{2 \mu_{e}}\left(\nabla_{1}^{2}+\nabla_{2}^{2}\right)-\frac{2 V_{0} \mathrm{e}^{-2 \alpha \eta_{1}}}{r_{1}}-\frac{2 V_{0}}{r_{1}}-2 V_{0} \alpha \mathrm{e}^{-2 \alpha \eta_{1}}\right. \\
& \left.-\frac{2 V_{0} \mathrm{e}^{-2 \alpha r_{2}}}{r_{2}}-\frac{2 V_{0}}{r_{2}}-2 V_{0} \alpha \mathrm{e}^{-2 \alpha r_{2}}-E\right) \psi\left(\boldsymbol{r}_{1}, \boldsymbol{r}_{2}\right)=0
\end{aligned}
$$




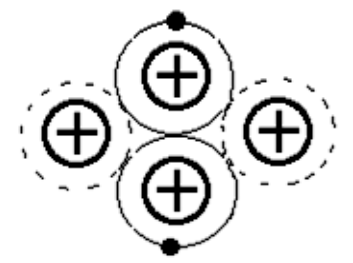

Figure 5. Helium nucleus with two protons and two neutrons.

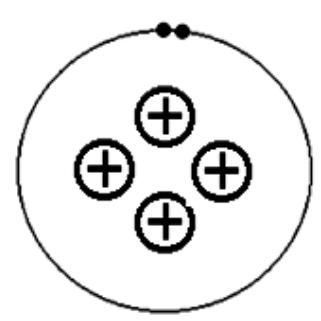

Figure 6. Helium nucleus with a generalised Yukawa potential.

Using the separable wavefunction $\psi\left(\boldsymbol{r}_{1}, \boldsymbol{r}_{2}\right)=\psi_{1}\left(\boldsymbol{r}_{1}\right) \psi_{2}\left(\boldsymbol{r}_{2}\right)$ the Schrödinger wave equation given in Equation (27) can now be written as a system of two separate Schrödinger wave equations

$$
\begin{gathered}
\left(-\frac{\hbar^{2}}{2 \mu_{e}} \nabla_{1}^{2}-\frac{2 V_{0} \mathrm{e}^{-2 \alpha r_{1}}}{r_{1}}-\frac{2 V_{0}}{r_{1}}-2 V_{0} \alpha \mathrm{e}^{-2 \alpha \eta_{1}}\right) \psi_{1}\left(\boldsymbol{r}_{1}\right)=E_{1} \psi_{1}\left(\boldsymbol{r}_{1}\right) \\
\left(-\frac{\hbar^{2}}{2 \mu_{e}} \nabla_{2}^{2}-\frac{2 V_{0} \mathrm{e}^{-2 \alpha r_{2}}}{r_{2}}-\frac{2 V_{0}}{r_{2}}-2 V_{0} \alpha \mathrm{e}^{-2 \alpha r_{2}}\right) \psi_{2}\left(\boldsymbol{r}_{2}\right)=E_{2} \psi_{2}\left(\boldsymbol{r}_{2}\right)
\end{gathered}
$$

where $E=E_{1}+E_{2}$. Solutions to Equations (28) and (29) are the radial eigenfunctions of the bound states which can also be obtained as

$$
R_{n l}\left(r_{i}\right)=N_{n l} r_{i}^{(-1+\sqrt{4 l(l+1)+1}) / 2} \mathrm{e}^{-\beta r} L_{n}^{1+\sqrt{4 l(l+1)+1}}\left(2 \beta r_{i}\right), i=1,2
$$

where $\beta^{2}=\left(2 \mu_{e}\left(V_{0}+V_{0} \mathrm{e}^{-2 \alpha r_{i}}\right) / \hbar^{2}(2 n+1+\sqrt{4 l(l+1)+1})\right)^{2}$. The corresponding energy spectrum $E_{n l}^{i}$ is given by

$$
E_{n l}^{i}=-V_{0} \alpha \mathrm{e}^{-2 \alpha r_{i}}-\frac{\mu_{e}}{2 \hbar^{2}}\left(\frac{\left(V_{0}+V_{0} \mathrm{e}^{-2 \alpha r_{i}}\right)}{n+l+1}\right)^{2}, i=1,2
$$

Similar to the case discussed in Section 2, the generalised Yukawa MGESCP potential given in Equation (1) is reduced to the potential that is used to describe the strong force

$$
\begin{aligned}
V\left(r_{i}\right) & =-\frac{V_{0}}{r_{i}}\left(1+\left(1+\alpha r_{i}\right) \mathrm{e}^{-2 \alpha r}\right) \\
& =-\frac{V_{0}}{r_{i}}+\frac{k J}{2 \alpha}\left(1+\alpha r_{i}\right) \\
& =-\frac{V_{0}}{r_{i}}+\frac{k J}{2} r_{i}+\frac{k J}{2 \alpha}
\end{aligned}
$$


And the corresponding energy spectrum can be obtained as

$$
E_{n l}^{i}=-\frac{k J}{2} r_{i}-\frac{\mu_{e}}{2 \hbar^{2}}\left(\frac{\left(V_{0}+(k J \alpha / 2) r_{i}\right)}{n+l+1}\right)^{2}, i=1,2
$$

Now, we extend our discussion on molecular structure of atomic nucleus to nuclei of higher number of protons and neutrons. Such possible molecular structures are shown in the following figures for nuclei with even numbers of protons and neutrons, respectively. There is a new feature in these figures representing by the symbol $\bigcirc$, which is a speculative neutral element that may help keep the nuclei in a more stable structure. In this work we only investigate possible molecular structures formed by protons and neutrons therefore the questions about the existence as well as the nature of these neutral particles are not essential and required further investigations.

In Figures 7-9, each pair of neutrons next to each other share a pair of electrons and each proton shares a pair of electrons given by the two neutrons next to it. They may be considered as the net of a polyhedron. As shown in Figure 8 and Figure 9, despite both nuclei have the same number of protons and neutrons, they may have different net polyhedrons due to the existence of different neutral particles inside the nuclei. With regard to rigorous mathematical formulation, it is worth mentioning here that the above molecular structures of atomic nuclei may also be determined geometrically and topologically by moduli spaces of circle packings [11] [12] [13].

For the case of nuclei with odd numbers of protons and neutrons, we suggest that they would have a molecular structure which is a coupling of a deuteron and another nucleus of even numbers of protons and neutrons. For example, in Figure 10 the nucleus is a coupling of the nucleus a beryllium and a deuteron. Therefore, in the case of nuclei with odd numbers of protons and neutrons, besides atomic bonding we also have molecular bonding between the nuclei which are now have molecular structures.

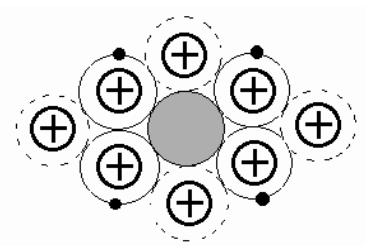

Figure 7. Nucleus with four protons and four neutrons.

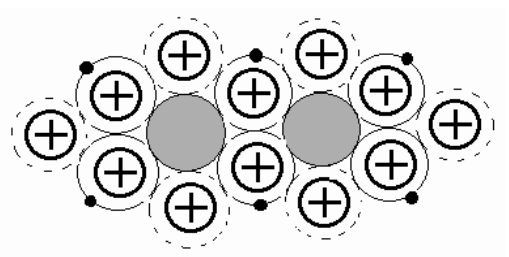

Figure 8. Nucleus with six protons and six neutrons. 


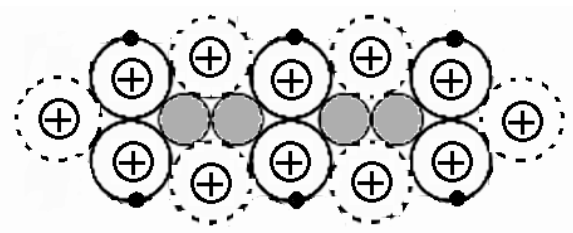

Figure 9. Nucleus with six protons and six neutrons.

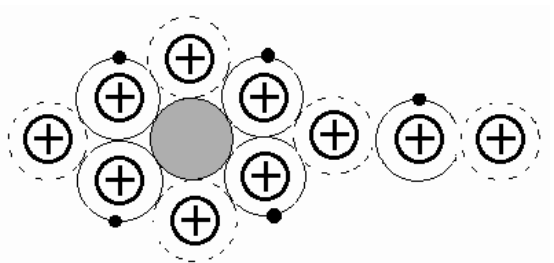

Figure 10. Nucleus with five protons and five neutrons.

The molecular structures of nuclei with many protons and neutrons that are depicted in the above suggestive figures are long and narrow lattices therefore in order to form spherical shapes, that they are assumed to possess, they should fold to form three-dimensional spherical structures by internal bonding similar to hydrogen bonding. On the other hand, even though it may seem highly speculative, it is possible to suggest that the three-dimensional molecular structures may also form layers of shells with the characteristics that have been studied in nuclear physics, such as the nucleons of an atomic nucleus may form shells similar to the shell model of an atom [14] [15] [16]. However, the nuclear shell model is based on an informative experimental observation that a nucleus is more stable if it has the so-called magic numbers of nucleons, which are 2, 8, 20, $28,50,82$ and 126 . What is the physical, as well as mathematical, basis for these magic numbers? Do they reflect a physical pattern that is geometrically associated with the distribution? In the following we will discuss how these numbers may in fact be associated with a more subtle structure of the nucleons based on the Heisenberg model of the neutron. As in the case of solid formed by normal atoms, it is assumed that the valence electrons can move freely around all atoms. We now also assume that the valence electrons released from the neutrons can also move freely around all protons in the lattice. In this case when a nucleus is formed it can be formed in the shape of spherical objects. Furthermore, we may also suggest that the structures formed by the nucleons have stable mathematical structures such as that of fullerenes [17] [18], in which the vertices are occupied by the nucleons which are now simply just protons. For example, for the case of nuclei with many neutrons and protons they may arrange themselves into the structures of buckminsterfullerene as follows.

The buckminsterfullerene structure of a nucleus given in Figure 11 can be identified with that of germanium if we assume it has the magic number of 28 neutrons and 32 protons. We now raise the question of what force would be required for the nucleons to be arranged in such shape. As in the case of normal 


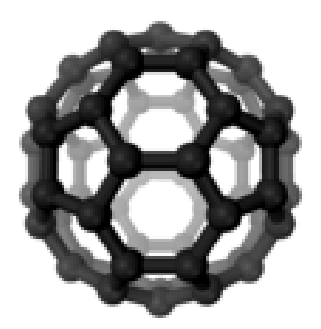

Figure 11. Buckminsterfullerene structure of a nucleus.

metals, we may assume that the nucleons are held together by delocalised bonds and the electrons are shared by all protons resulting positive ions being surrounded by a cloud of electrons.

Similar to the case of the nucleus of a helium atom discussed above, if we assume the protons to be arranged into a spherical structure such as that given in the Figure 11 so that the electron cloud can form shells just above the surface of the protons. The quantum dynamics of such physical system can be described by the Schrödinger wave equation using the central field approximation. Consider a nucleus of charge $Z q$ and $N$ electrons. Assume the part of the nucleus composed of the protons to be point-like and infinitely massive. If we only consider the electrostatic Coulomb repulsion between the electrons, the generalised Yukawa interaction between the electrons and the protons then the Hamiltonian of the $\mathrm{N}$-neutron atomic nucleus can be written as

$$
H=\sum_{i=1}^{N}\left(-\frac{\hbar^{2}}{2 \mu_{e}} \nabla_{r_{i}}^{2}-\frac{2 V_{0} \mathrm{e}^{-2 \alpha r_{i}}}{r_{i}}-\frac{2 V_{0}}{r_{i}}-2 V_{0} \alpha \mathrm{e}^{-2 \alpha r_{i}}\right)+\sum_{i<j=1}^{N} \frac{q^{2}}{4 \pi \varepsilon_{0} r_{i j}}
$$

Again, if we assume the term associated with the Coulomb force between the electrons can be neglected then the Schrödinger wave equation for the spatial part of the $N$ electrons can be written as

$$
\left(\sum_{i=1}^{N}\left(-\frac{\hbar^{2}}{2 \mu_{e}} \nabla_{r_{i}}^{2}-\frac{2 V_{0} \mathrm{e}^{-2 \alpha r_{i}}}{r_{i}}-\frac{2 V_{0}}{r_{i}}-2 V_{0} \alpha \mathrm{e}^{-2 \alpha r_{i}}\right)-E\right) \psi\left(\boldsymbol{r}_{1}, \cdots, \boldsymbol{r}_{N}\right)=0
$$

This equation is separable into $N$ equations each of which describes the dynamics of one electron. In this case the solution $\psi\left(\boldsymbol{r}_{1}, \cdots, \boldsymbol{r}_{N}\right)$ may be written as $\psi\left(\boldsymbol{r}_{1}, \cdots, \boldsymbol{r}_{N}\right)=\psi_{1}\left(\boldsymbol{r}_{1}\right) \psi_{2}\left(\boldsymbol{r}_{2}\right) \cdots \psi_{n}\left(\boldsymbol{r}_{N}\right)$ and each wavefunction $\psi_{i}\left(\boldsymbol{r}_{i}\right)$ satisfies the equation

$$
\left(-\frac{\hbar^{2}}{2 \mu_{e}} \nabla_{i}^{2}-\frac{2 V_{0} \mathrm{e}^{-2 \alpha r_{i}}}{r_{i}}-\frac{2 V_{0}}{r_{i}}-2 V_{0} \alpha \mathrm{e}^{-2 \alpha r_{i}}\right) \psi_{i}\left(\boldsymbol{r}_{\boldsymbol{i}}\right)=E_{i} \psi_{i}\left(\boldsymbol{r}_{i}\right)
$$

where $E=E_{1}+E_{2}+\cdots+E_{N}$. Solutions to Equation (36) and the corresponding generalised Yukawa MGESCP potential and energy spectrum can be obtained similar to those given in Equations (30-33) with $i=1,2, \cdots, N$.

\section{Conclusion}

We have investigated and shown in this work that it is possible to apply the Hei- 
senberg model of the neutron formulated as a dwarf hydrogen-like atom into the construction of molecular structure for an atomic nucleus in which the nucleus would possess the structure of a molecule made up of atoms bonding together by a generalised Yukawa MGESCP potential used to describe the strong force. First, we construct the molecular structure for the atomic nucleus of the deuterium and show that such molecular structure would possess similar formation to that of the hydrogen molecular ion $\mathrm{H}_{2}^{+}$which has only one electron orbiting two protons under a Yukawa MGESCP potential instead of the Coulomb potential. From this foundational approach, we then extend our discussion into the construction of molecular structure for nuclei composed of many protons and neutrons, and we show that the protons and neutrons of these nuclei can also be assembled to possess a molecular structure if the neutrons are regarded as dwarf hydrogen atoms. We also show that it is possible to apply Schrödinger wave equation with central field approximation to describe the quantum dynamics of nuclei of atomic atoms that now possess the physical structure of dwarf molecular ions under a Yukawa MGESCP potential. As a further discussion, we then suggest that the structures formed by the nucleons may have stable mathematical structures such as that of fullerenes in which the vertices are occupied by just protons. For example, a nucleus with a total number of 60 nucleons may arrange itself into the topological structure of a buckminsterfullerene.

\section{Acknowledgements}

We would like to thank the reviewers for their constructive comments and we would also like to thank Jane Gao of the administration of JMP for her editorial advice during the preparation of this work.

\section{Conflicts of Interest}

The author declares no conflicts of interest regarding the publication of this paper.

\section{References}

[1] Ho, V.B. (2019) Journal of Modern Physics, 10, 1065-1082. https://doi.org/10.4236/jmp.2019.109069

[2] Ikhdair, S.M. and Sever, R. (2016) Journal of Mathematical Chemistry, 41, 343-353. https://doi.org/10.1007/s10910-007-9226-X

[3] Ita, B.I., Louis, H., Akakuru, O.U., Magu, T.O., Joseph, I., Tchoua, P., Amos, P.I., Effiong, I. and Nzeata, N.A. (2018) Journal of Quantum Information Science, 8 24-45. https://doi.org/10.4236/jqis.2018.81003

[4] Ho, V.B. (2016) Formulating Physics Purely in Terms of Differential Geometry and Topology. Project.

[5] Schrödinger, E. (1982) Collected Papers on Wave Mechanics. AMS Chelsea Publishing, New York.

[6] Schrödinger, E. (1926) Physical Review, 28, 1049-1070.

https://doi.org/10.1103/PhysRev.28.1049 
[7] Dirac, P.A.M. (1928) Proceedings of the Royal Society A: Mathematical, Physical and Engineering Sciences, 117, 610-624. https://doi.org/10.1098/rspa.1928.0023

[8] Ho, V.B. (2020) Classification of Relativity. Journal of Modern Physics, 11, 535-564. https://doi.org/10.4236/jmp.2020.114036

[9] Bransden, B.H. and Joachain, C.J. (1989) Introduction to Quantum Mechanics. Longman Scientific \& Technical, New York.

[10] Bransden, B.H. and Joachain, C.J. (1983) Physics of Atoms and Molecules. Longman Scientific \& Technical, New York.

[11] Hatcher, A. and Thurston, W. (2015) Moduli Spaces of Circle Packings.

[12] Stephenson, K. (2003) Notices of the American Mathematical Society, 50, 1376-1388.

[13] Stephenson, K. (2004) Introduction to Circle Packing and the Theory of Discrete Analytic Function. Cambridge University Press, Cambridge.

[14] Bethe, H.A. and Morrison, P. (2006) Elementary Nuclear Theory. Dover Publications, Inc., New York.

[15] Wong, S.S.M. (2004) Introductory Nuclear Physics. Wiley, Hoboken.

[16] Basdevant, J.-L., Rich, J. and Spiro, M. (2004) Fundamentals in Nuclear Physics. Springer, New York.

[17] Schwerdtfeger, P., Wirz, L. and Avery, J.E. (2014) Wiley Interdisciplinary Reviews. Computational Molecular Science, 5, 96-145. https://doi.org/10.1002/wcms.1207

[18] Fullerene, Encyclopaedia Britannica Online. 